
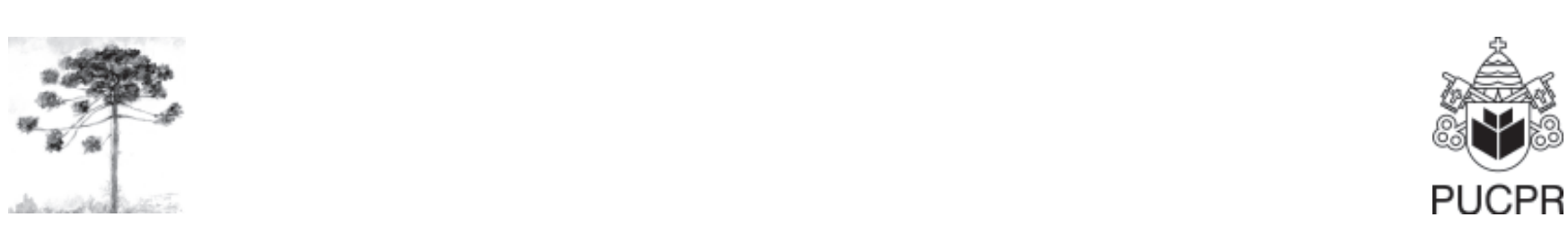

\title{
EFEITO DA TEMPERATURA E DO FOTOPERÍODO NO CRESCIMENTO E NAS RESPOSTAS FOTOSSINTÉTICAS DE Potamogeton pectinatus L. (POTAMOGETONACEAE), EM CULTIVO EXPERIMENTAL ${ }^{1}$
}

\author{
Effects of temperature and photoperiod on growth and photosynthetic responses of \\ Potamogeton pectinatus L. (Potamogetonaceae), under controlled culture conditions
}

\author{
Ioni Gonçalves Colares ${ }^{[1]}$, Thasiana de Lima Batista ${ }^{[2]}$, Ubiracy Allan Sousa Magalhães ${ }^{[2]}$, \\ Luciane Cougo dos Santos ${ }^{[3]}$, Mirian Daniela Buchweitz Schlee ${ }^{[4]}$
}

\footnotetext{
${ }^{[1]}$ Setor de Botânica, Instituto de Ciências Biológicas, Universidade Federal do Rio Grande (FURG), Rio Grande, RS - Brasil, e-mail:dmbioni@furg.br

${ }^{[2]}$ Mestrandos em Biologia de Ambientes Aquáticos Continentais, Universidade Federal do Rio Grande (FURG), Rio Grande, RS - Brasil.

${ }^{[3]}$ Acadêmica do Curso de Ciências Biológicas, Bolsista de Iniciação Científica CNPq, Universidade Federal do Rio Grande (FURG), Rio Grande, RS - Brasil.

${ }^{[4]}$ Acadêmica do Curso de Ciências Biológicas, Universidade Federal do Rio Grande (FURG), Rio Grande, RS - Brasil.
}

\section{Resumo}

Macrófitas submersas de regiões temperadas apresentam marcada sazonalidade no crescimento e biomassa por causa das trocas nas condições ambientais. $\mathrm{O}$ objetivo deste estudo foi avaliar, em cultivo experimental, o efeito da temperatura e do fotoperíodo no crescimento e nas respostas fotossintéticas de Potamogeton pectinatus L., coletada na Lagoa Verde. As plantas foram cultivadas em diferentes temperaturas e fotoperíodos, sendo o crescimento avaliado utilizando o número de folhas, de raízes e a biomassa. As respostas fotossintéticas foram avaliadas pelo método de evolução do oxigênio, montando curvas PI em diferentes temperaturas e fotoperíodos. A produção de folhas $\left(30 \pm 2,2\right.$ folhas) foi significativamente maior $(\mathrm{p}<0,05)$ na temperatura de $30^{\circ} \mathrm{CD} / 25^{\circ} \mathrm{CN}$, fotoperíodo de $10 \mathrm{hL} / 14 \mathrm{hE}$. Plantas mantidas em baixa temperatura $\left(10^{\circ} \mathrm{CD} / 05^{\circ} \mathrm{CN}\right)$ apresentaram uma produção de folhas reduzida nos três fotoperíodos testados, investindo no alongamento das já existentes. As

${ }_{1}$ Financiamento: Fundação de Assistência à Pesquisa do Estado do Rio Grande do Sul (FAPERGS). 
maiores taxas de crescimento específico foliar foram observadas na temperatura baixa de $10^{\circ} \mathrm{CD} /$ $05^{\circ} \mathrm{CN}$. Valores de Pmax foram crescentes com o aumento do fotoperíodo em temperaturas de crescimento baixas $\left(10^{\circ} \mathrm{CD} / 05^{\circ} \mathrm{CN}\right)$ e decrescentes nas temperaturas altas $\left(30^{\circ} \mathrm{CD} / 25^{\circ} \mathrm{CN}\right)$, com maiores valores em $20^{\circ} \mathrm{CD} / 15^{\circ} \mathrm{C}$. O maior valor de a foi observado em plantas crescendo em temperaturas de $20^{\circ} \mathrm{CD} / 15^{\circ} \mathrm{CN}$, fotoperíodo de $10 \mathrm{hL} / 14 \mathrm{hE}\left(\mathrm{a}=0,92 \mathrm{gO}_{2} \mathrm{gMS}^{-1} \mathrm{~h}^{-1} / \mathrm{mmol} \mathrm{m}^{-2} \mathrm{~s}^{-1}\right)$. Plantas crescendo na temperatura de $30^{\circ} \mathrm{CD} / 25^{\circ} \mathrm{CN}$, fotoperíodo $14 \mathrm{hL} / 10 \mathrm{hE}$ apresentaram valores elevados de Ic e Ik. As respostas fotossintéticas e o crescimento, apresentados por P. pectinatus neste estudo, mostram a plasticidade morfológica e fisiológica das plantas para sobreviver e se desenvolver nas diferentes condições sazonais de temperatura e fotoperíodo.

Palavras-chave: Potamogeton pectinatus; Fotossíntese; Crescimento; Fotoperíodo; Temperatura.

\begin{abstract}
Submerged macrophytes in temperate regions show marked seasonality in growth and biomass due to trade in environmental conditions. This study aimed to evaluate in experimental culture, the effect of temperature and photoperiod on growth and photosynthetic responses of Potamogeton pectinatus L., collected in Lagoa Verde lake. The plants were grown at different temperatures and photoperiods, the growth is evaluated using the number of leaves, roots and biomass. The photosynthetic responses were evaluated by the method of oxygen evolution, up PI curves at different temperatures and photoperiods. The production of leaves $(30 \pm 2.2)$ was significantly higher $(P<0.05)$ in the temperature of $30^{\circ} \mathrm{CD} / 25^{\circ} \mathrm{CN}$, photoperiod of $10 \mathrm{hL} / 14 \mathrm{hE}$. Plants kept at low temperature $\left(10^{\circ} \mathrm{CD} / 05^{\circ} \mathrm{CN}\right)$ showed a reduced production of leaves in the three photoperiods tested, investing in the elongation of existing ones. The highest growth rates specific leaves were observed in temperature of $10^{\circ} \mathrm{CD} / 05^{\circ} \mathrm{CN}$. Pmax values were increased with increasing photoperiod in the growth of low temperatures $\left(10^{\circ} \mathrm{CD} / 05^{\circ} \mathrm{CN}\right)$ and decreasing the high temperatures $\left(30^{\circ} \mathrm{CD} / 25^{\circ} \mathrm{CN}\right)$, with higher values was observed in plants at $20^{\circ} \mathrm{C} /$ $15^{\circ} \mathrm{CD}$. The greatest value of a growing in temperatures of $20^{\circ} \mathrm{CD} / 15^{\circ} \mathrm{CN}$, photoperiod of $10 \mathrm{hL} / 14 \mathrm{hE}\left(a=0.92 \mathrm{gO}_{2} \mathrm{gDW}^{-1} \mathrm{~h}^{-1} / \mathrm{mmol} \mathrm{m}^{-2} \mathrm{~s}^{-1}\right)$. Plants growing in temperature of $30^{\circ} \mathrm{CD} / 25^{\circ} \mathrm{CN}$, photoperiod $14 \mathrm{hL} / 10 \mathrm{hE}$ showed high values of Ic and Ik. Responses photosynthesis and growth, presented by P. pectinatus in this study, show the morphological and physiological plasticity of plants to survive and develop in different seasonal conditions of temperature and photoperiod.
\end{abstract}

Keywords: Potamogeton pectinatus; Growth; Photosynthesis; Photoperiod; Temperature.

\section{INTRODUÇÃO}

Ecossistemas de macrófitas aquáticas ocupamimportante papel como produtores primários em rios e lagoas, fornecendo hábitats e áreas de crescimento e desenvolvimento para muitos organismos aquáticos. Separações temporais no crescimento e biomassa, bem como suas respostas fotossintéticas podem ser reguladas pela variação na temperatura e irradiância $(1,2)$.

Flutuações na irradiação subaquática fazem com que as macrófitas submersas necessitem de contínuos ajustes fisiológicos para manter seu metabolismo positivo (3). Variações temporais resultam em modelos diferenciais de utilização de recursos para o crescimento, com melhor desempenho quanto maior a temperatura e quantidade de radiação recebida, resultando em estações de crescimento características (4). Ambientes aquáticos podem modificar alguns aspectos da luz incidente, onde o aumento na profundidade da água, alterando o comprimento do fotoperíodo, pode interferir na absorção de nutrientes e consequentemente no crescimento das plantas (5-7).

A habilidade para se desenvolvere sobreviver em diferentes condições termais envolve muitos processos integrados da planta (8) e a capacidade de 
aclimatação às variações na temperatura podem aumentar sua produtividade (9). Temperaturas ótimas para os processos biológicos são variáveis, ocorrendo queda na produtividade quando a temperatura se distancia do ótimo (10). Ajustes morfológicos ou mudanças no modelo de alocação de biomassa também podem melhorar o desempenho da planta sob diferentes regimes termais (8).

Potamogeton pectinatus L., família Potamogetonaceae, é uma macrófita submersa, de regiões temperadas, com ampla distribuição pelos sistemas de rios, lagoas e áreas costeiras de baixa salinidade. Possui as folhas alongadas e finas, com os ramos delgados e perenes, com crescimento apical, desenvolvendo abundante biomassa estratificada quando as condições ambientais são favoráveis (11). Pode apresentar diferentes ciclos de vida (12), sendo a principal forma de reprodução e distribuição por meio de tubérculos subterrâneos ou fragmentação dos rizomas $(13,14)$. O predomínio da reprodução assexuada facilita o cultivo da espécie em condições de laboratório. A importância desses estudos em laboratório está na manipulação de diferentes fatores, e seus efeitos sobre a fisiologia e ecologia da espécie em questão (15). Medidas de fotossíntese in situ são logisticamente difíceis de serem realizadas (16). Parâmetros fotossintéticos são normalmente estimados em incubações em laboratório sob diferentes condições de intensidade luminosa (10). Sendo assim, o objetivo deste trabalho foi avaliar o efeito da temperatura e do fotoperíodo no crescimento e nas respostas fotossintéticas de Potamogeton pectinatus L., em cultivo experimental.

\section{MATERIAL E MÉTODOS}

\section{Coleta e cultivo das plantas}

As plantas de $P$. pectinatus, utilizadas neste estudo, foram coletadas na Lagoa Verde, situada em $32^{\circ} 07.923^{\prime}$ S e $52^{\circ} 10.858^{\prime} \mathrm{W}$, no município de Rio Grande, RS, Brasil. O clima na região é temperado quente com invernos frios e chuvosos e verões secos e quentes. A profundidade média da coluna d'água na Lagoa Verde é de 47,3 $\pm 10,5 \mathrm{~cm}$, com temperatura média anual da água de $22,4 \pm 2,8^{\circ} \mathrm{C}(12)$.

As plantas coletadas foram acondicionadas em béckers de $3000 \mathrm{~mL}$, preenchidos com água da Lagoa Verde, filtrada com filtro Whatman $\mathrm{n}^{\circ} 3$, utilizando cinco repetições, e mantidas em incubadora com padrões de temperatura diurna (D) e noturna $(\mathrm{N})$ específicos para cada teste. O fotoperíodo foi mantido usando lâmpadas fluorescentes brancas frias como fonte de luz, posicionadas lateralmente aos frascos de cultivo com irradiância constante de $230 \mathrm{mmolm}^{-2} \mathrm{~s}^{-1}$. A água dos béckers foi trocada a cada três dias, de modo a garantir nutrientes suficientes para o crescimento.

\section{Crescimento}

Para definir a longevidade foliar, cinco plantas com tamanho e número de folhas similares foram transferidas para béckers de $500 \mathrm{~mL}$, sendo submetidas a seis padrões de temperatura: $30^{\circ} \mathrm{CD}$ $25^{\circ} \mathrm{CN}, 25^{\circ} \mathrm{CD}-20^{\circ} \mathrm{CN}, 20^{\circ} \mathrm{CD}-15^{\circ} \mathrm{CN}, 15^{\circ} \mathrm{CD}-$ $10^{\circ} \mathrm{CN}, 10^{\circ} \mathrm{CD}-05^{\circ} \mathrm{CN}, 05^{\circ} \mathrm{CD}-0^{\circ} \mathrm{CN}$, fotoperíodo $12 \mathrm{hL} / 12 \mathrm{hE}$. A longevidade foliar, em cada repetição, foi medida com base no tempo decorrido entre a produção da primeira folha, marcada com caneta de tinta à prova d'água, até a sua morte (folhas brancas-amareladas).

A seguir, para testar o efeito conjugado da temperatura e do fotoperíodo sobre o crescimento, foram fixadas três temperaturas: alta $\left(30^{\circ} \mathrm{CD}-25^{\circ} \mathrm{CN}\right)$, intermediária $\left(20^{\circ} \mathrm{CD}-15^{\circ} \mathrm{CN}\right)$ e baixa $\left(10^{\circ} \mathrm{CD}-05^{\circ} \mathrm{C}\right)$, nos fotoperíodos: $10 \mathrm{hL} /$ 14hE, $12 \mathrm{hL} / 12 \mathrm{hE} \mathrm{e} 14 \mathrm{hL} / 10 \mathrm{hE}$, totalizando nove variações. Após 10 dias de aclimatação, em cada condição experimental, foram avaliados:

a) O número de folhas e raízes novas produzidas diariamente em cada repetição, durante 30 dias;

b) O comprimento final alcançado pelas folhas e raízes ao final do experimento;

c) O acréscimo de biomassa aérea (folhas) e subterrânea (raízes), durante o período de crescimento, foi obtido a partir da massa de matéria seca, após secagem em estufa a $80^{\circ} \mathrm{C}$.

A taxa de crescimento específico foi calculada pela equação: $\mathrm{m}=\left(\ln \mathrm{B}_{\mathrm{t}}-\ln \mathrm{B}_{\mathrm{o}}\right) \cdot \mathrm{t}^{-1}$, onde: $\mathrm{B}$ é a quantidade de biomassa inicial produzida e $\mathrm{B}_{\mathrm{t}}^{\mathrm{o}}$, a quantidade de biomassa final, após t dias de crescimento $(17,18)$. A quantidade de biomassa inicial das folhas utilizadas nos experimentos foi estimada a partir da equação de regressão entre o 
comprimento foliar e a massa de matéria seca, de 60 folhas medidas independentemente. A taxa de crescimento específico foi expressa em $\mathrm{gd}^{-1} \mathrm{MS}$ (gramas por dia de matéria seca).

\section{Respostas fotossintéticas}

Odesempenho fotossintético de P.pectinatus foi determinado através da técnica de evolução do oxigênio dissolvido. Para efeito de padronização utilizaram-se apenas as folhas da região apical dos rizomas. Os experimentos foram realizados em garrafas de DBO de $300 \mathrm{ml}$, preenchidas com água da Lagoa Verde, filtrada com filtro Whatman $n^{\circ} 3$. As garrafas foram mantidas submersas em uma câmara de incubação com água circulante, nas temperaturas de $30^{\circ} \mathrm{C}, 20^{\circ} \mathrm{C}$ e $10^{\circ} \mathrm{C}$, por duas horas. A fonte de luz utilizada foi uma lâmpada de halogênio marca Osram (1000 Watts) e as radiações necessárias para as relações de fotossíntese $\mathrm{x}$ irradiância $(0,15$, 30, 60, 100, 500, 1000, $2900 \mu \mathrm{mol} \mathrm{m} \mathrm{m}^{-1}$ ) foram obtidas cobrindo as garrafas com filtros de tela preta. As radiações fotossinteticamente ativas (PAR) foram medidas com o aparelho Li-Cor Radiation Sensor LI-1400 Datalogger, sendo utilizadas quatro réplicas para cada intensidade luminosa. Leituras de concentração de oxigênio dissolvido antes e após a incubação, em cada amostra, foram feitas utilizando o eletrodo tipo Digimed DMO-2.

Os dados foram agrupados de maneira a obter curvas PI (regressões de fotossíntese $\mathrm{x}$ irradiância), plotadas para diferentes temperaturas e fotoperíodos. As medidas de fotossíntese líquida e a respiração foram determinadas utilizando as equações de Stricland \& Parsons (19). Foram determinados cinco parâmetros da curva PI (20): 1) A respiração foi determinada como a média do consumo de oxigênio no escuro. 2) A fotossíntese líquida máxima (Pmax) foi determinada pela evolução de oxigênio nas radiações de incubação de 500, 1000 e 2900 mmol m $\mathrm{m}^{-1}$ (21). 3) Uma regressão linear usando as taxas fotossintéticas nas radiações de $0,15,30 \mathrm{mmol}$ $\mathrm{m}^{-2} \mathrm{~s}^{-1}$ foi usada para calcular a inclinação inicial a (22). 4) A radiação de compensação (Ic), na qual a fotossíntese se iguala à respiração também foi determinada utilizando a mesma equação da regressão linear de a. 5) A radiação de saturação da fotossíntese (Ik) foi definida pela interseção entre as linhas criadas pela inclinação inicial (a) e o Pmax (23). As curvas PI foram padronizadas pelo peso de matéria seca das folhas. Ao final de cada curva PI, as folhas foram retiradas, pesadas, secas em estufa a $80^{\circ} \mathrm{C}$, por 72 horas. Os valores de peso de matéria seca foram utilizados para expressar a fotossíntese líquida em $\mathrm{mgO}_{2} \mathrm{gMS}^{-1} \mathrm{~h}^{-1}$.

\section{Análises estatísticas}

Para testar a hipótese de influência da temperatura e do fotoperíodo sobre a biomassa, produção de folhas e de raízes, comprimento foliar, taxa de crescimento específico, respostas fotossintéticas e parâmetros das curvas PI de $P$. pectinatus em laboratório, foram utilizadas ANOVA de duas vias, após a normalização dessas variáveis pela expressão: raiz quadrada de x. Para esta análise, utilizou-se o número de folhas e de raízes iniciais como covariáveis, a ser descontadas da análise (24). Os testes de ANOVAS foram seguidos de análises de contrastes, pelo teste de comparação múltipla de Tukey a 5\% de significância (24).

\section{RESULTADOS}

Maior longevidade foliar $(27,1 \pm 4,0$ dias $)$ foi alcançada em plantas crescendo nas temperaturas de $25^{\circ} \mathrm{CD}-20^{\circ} \mathrm{CN}$, fotoperíodo $12 \mathrm{hL}-12 \mathrm{hE}$, com as folhas alongando até a idade $11 \pm 6,9$ dias (Figura 1). Plantas crescendo em temperaturas de $05^{\circ} \mathrm{CD}$ $0^{\circ} \mathrm{CN}$ mostraram velocidade de crescimento mais lento até a idade de 12 dias, quando então começaram a desenvolver, embora atingindo um tamanho menor do que nas demais temperaturas (Figura 1). Plantas mantidas na temperatura $10^{\circ} \mathrm{CD} /$ $05^{\circ} \mathrm{CN}$, alcançaram o maior comprimento foliar $(4,1 \pm 0,42 \mathrm{~cm}$; Figura 2).

Ambos temperatura e fotoperíodo mostraram efeito significativo $(\mathrm{p}<0,05)$ no crescimento de $P$. pectinatus. A produção de folhas $(30 \pm 2,2$ folhas) foi significativamente maior $(\mathrm{p}<0,05)$ na temperatura de $30^{\circ} \mathrm{CD} / 25^{\circ} \mathrm{CN}$, fotoperíodo de $10 \mathrm{hL} / 14 \mathrm{hE}$ (Figura 2). Plantas mantidas em baixa temperatura $\left(10^{\circ} \mathrm{CD} / 05^{\circ} \mathrm{CN}\right)$ apresentaram produção de folhas reduzida nos três fotoperíodos testados (Figura 2), investindo mais no alongamento das já existentes (Figura 2). A produção de raízes não apresentou diferença significativa entre os diferentes experimentos $(\mathrm{p}>0,05)$. 


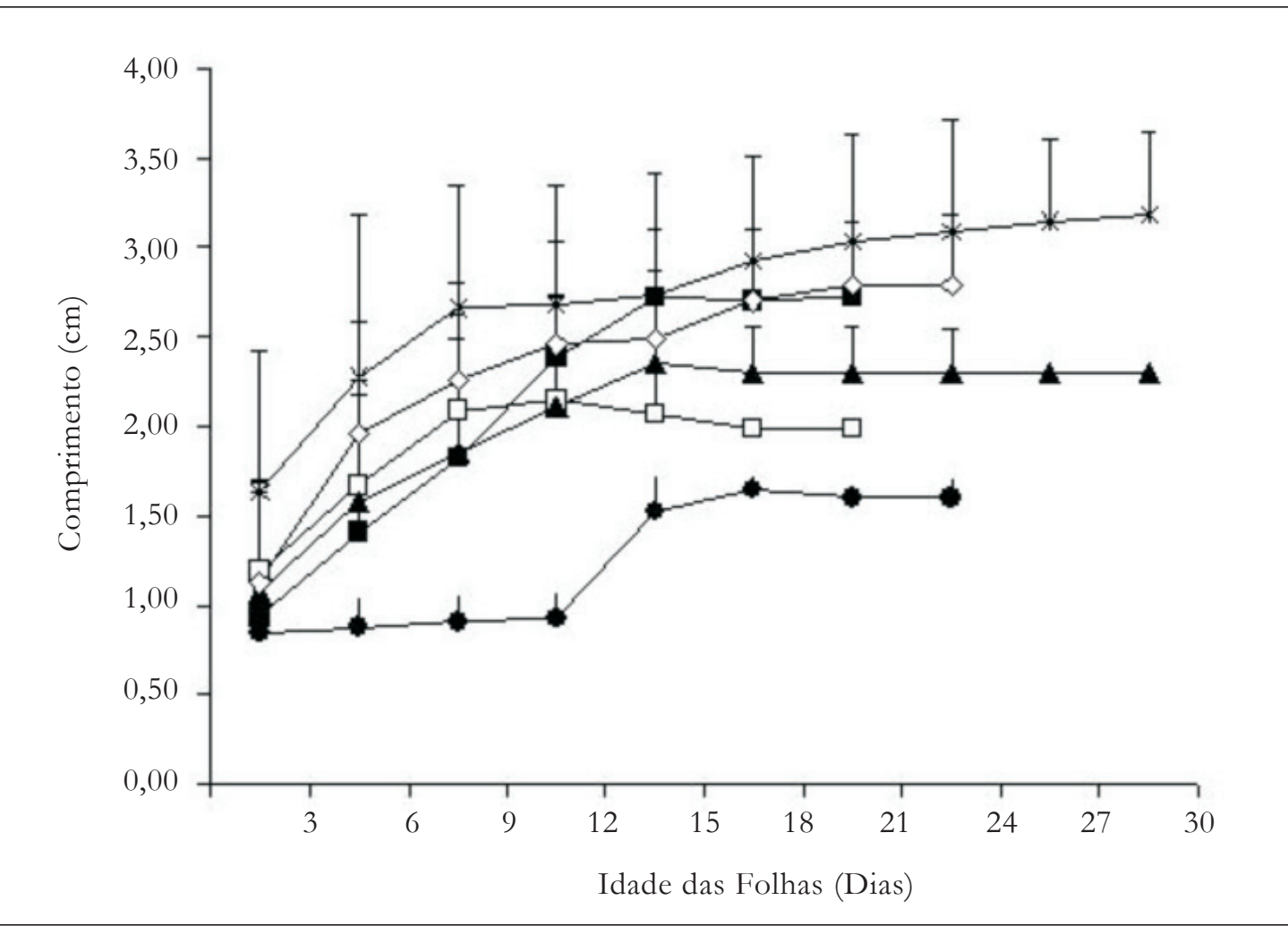

FIGURA 1 - Comprimento das folhas de Potamogeton pectinatus L. (médias \pm desvio-padrão), em função de sua idade, em plantas crescendo em cultivo experimental, sob diferentes temperaturas Nota: $05^{\circ} \mathrm{CD}-00^{\circ} \mathrm{CN} ; \quad 10^{\circ} \mathrm{CD}-05 \mathrm{CN} ; \quad 15^{\circ} \mathrm{CD}-10^{\circ} \mathrm{CN} ; \quad 20^{\circ} \mathrm{CD}-15 \mathrm{CN} ; \quad \mathbf{X} 25^{\circ} \mathrm{CD}-$ $20^{\circ} \mathrm{CN} ; 30^{\circ} \mathrm{CD}-25^{\circ} \mathrm{CN}$. (n $\left.=5\right)$

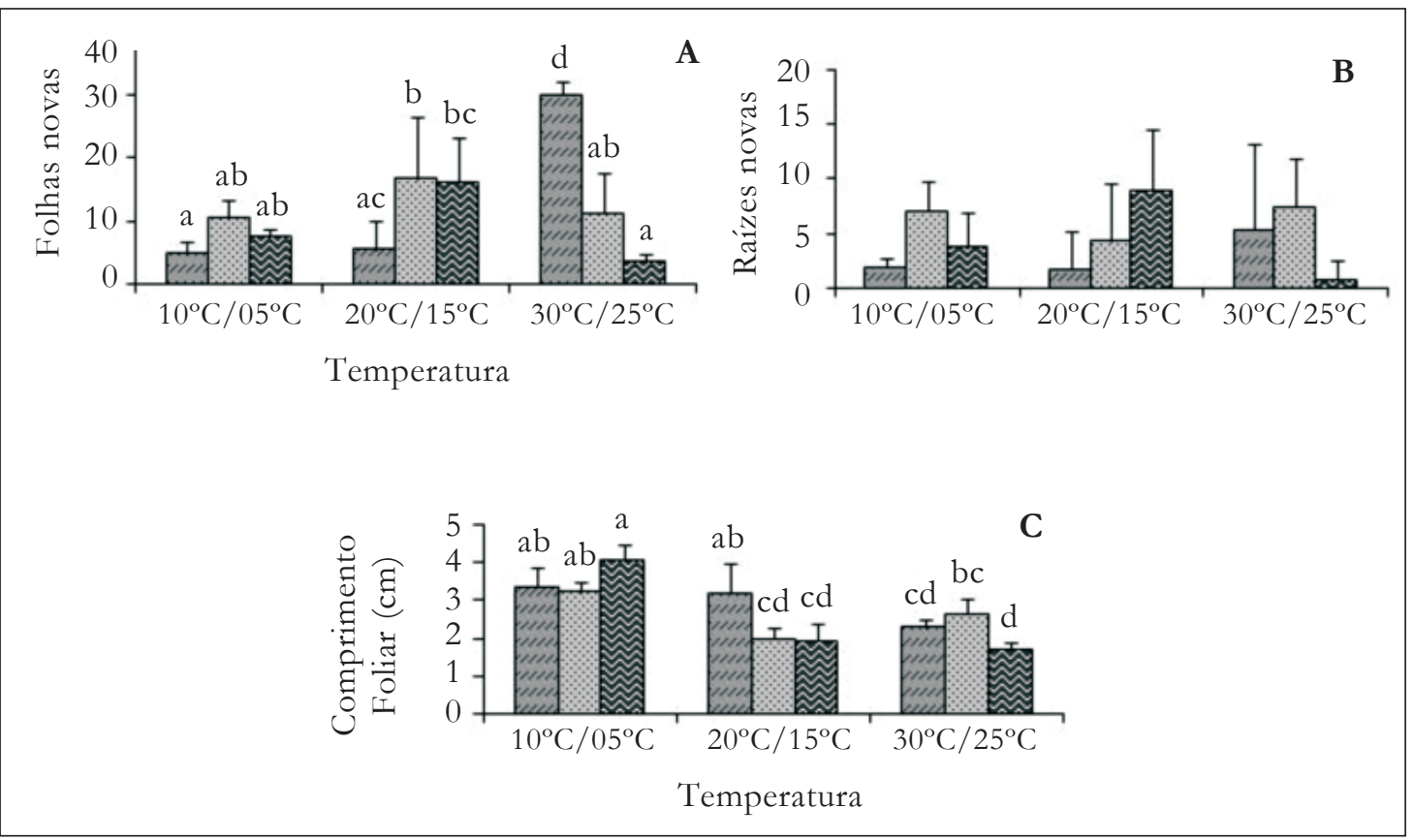

FIGURA 2 - Efeito da temperatura e fotoperíodo nas variáveis bióticas de plantas de Potamogeton pectinatus L. crescendo em cultivo experimental durante 30 dias (médias \pm desvio-padrão). Nota: Número de folhas novas produzidas (A), número de raízes novas produzidas (B) e comprimento foliar máximo (C). Fotoperíodos: 10hL/14hE; 12hL/12hE; $14 \mathrm{hL} /$ $10 \mathrm{hE}$. Letras iguais nas colunas indicam grupos homogêneos $(\mathrm{p}<0,05 ; \mathrm{n}=5)$ 
A biomassa aérea sofreu interferência do efeito conjugado da temperatura $\mathrm{x}$ fotoperíodo apenas na temperatura de $30^{\circ} \mathrm{CD} / 25^{\circ} \mathrm{CN}$, com respostas de crescimentos diferentes entre os fotoperíodos testados. $\mathrm{O}$ aumento do fotoperíodo nesta temperatura provocou uma queda significativa $(p<0,05)$ nos valores de biomassa aérea de 0,027 \pm 0,01gMS, em 10hL/14hE, para 0,009 \pm 0,003gMS, em 14hL/10hE (Tabela 1). A biomassa subterrânea respondeu positivamente ao aumento da temperatura, resultando na maior biomassa subterrânea $(0,05 \pm 0,03 \mathrm{gMS})$ no fotoperíodo de $12 \mathrm{hL} / 12 \mathrm{hE}$ à $30^{\circ} \mathrm{CD} / 25^{\circ} \mathrm{CN}(\mathrm{p}<0,05)$. As maiores taxas de crescimento específico foliar foram observadas na temperatura baixa de $10^{\circ} \mathrm{CD} / 05^{\circ} \mathrm{CN}$ (Tabela 1 ).

TABELA 1 - Biomassa da parte aérea (PA), subterrânea (PS), relação biomassa parte subterrânea / aérea (PS/PA) e taxa de crescimento específico das folhas de Potamogeton pectinatus, crescendo durante 30 dias em cultivo experimental sob diferentes temperaturas e fotoperíodos. $(n=5)$

\begin{tabular}{|c|c|c|c|c|c|}
\hline \multirow[b]{2}{*}{ Temp. } & \multirow[b]{2}{*}{ Fotop. } & \multicolumn{3}{|c|}{ Biomassa } & \multirow{2}{*}{$\begin{array}{c}\text { Taxa de } \\
\text { crescimento } \\
\text { específico }\end{array}$} \\
\hline & & Parte áerea & Parte subterrânea & PS/PA & \\
\hline${ }^{\circ} \mathrm{CD} /{ }^{\circ} \mathrm{CN}$ & $\mathrm{hL} / \mathrm{hE}$ & (g de MS) & (g de MS) & (g de MS) & $\left(\mathrm{g} \mathrm{dia}^{-1}\right)$ \\
\hline $10 / 05$ & $10 / 14$ & $0,012 \pm 0,004^{a}$ & $0,011 \pm 0,002^{\mathrm{a}}$ & $0,95 \pm 0,2^{\mathrm{a}}$ & $0,09 \pm 0,005^{a}$ \\
\hline $10 / 05$ & $12 / 12$ & $0,016 \pm 0,003^{\mathrm{ab}}$ & $0,02 \pm 0,002^{\mathrm{b}}$ & $0,81 \pm 0,1^{\mathrm{ab}}$ & $0,09 \pm 0,002^{\mathrm{ab}}$ \\
\hline $10 / 05$ & $14 / 10$ & $0,02 \pm 0,005^{\mathrm{ab}}$ & $0,019 \pm 0,004^{\mathrm{b}}$ & $0,88 \pm 0,1^{\mathrm{ab}}$ & $0,08 \pm 0,005^{a}$ \\
\hline $20 / 15$ & $10 / 14$ & $0,014 \pm 0,003^{\mathrm{a}}$ & $0,012 \pm 0,001^{\mathrm{a}}$ & $0,92 \pm 0,4^{\mathrm{a}}$ & $0,06 \pm 0,01^{\mathrm{ab}}$ \\
\hline $20 / 15$ & $12 / 12$ & $0,02 \pm 0,01^{\mathrm{b}}$ & $0,015 \pm 0,009^{\mathrm{ab}}$ & $0,98 \pm 0,8^{\mathrm{ab}}$ & $0,08 \pm 0,02^{\mathrm{a}}$ \\
\hline $20 / 15$ & $14 / 10$ & $0,019 \pm 0,005^{\mathrm{ab}}$ & $0,019 \pm 0,005^{\mathrm{b}}$ & $1,11 \pm 0,5^{\mathrm{ab}}$ & $0,08 \pm 0,02^{\mathrm{b}}$ \\
\hline $30 / 25$ & $10 / 14$ & $0,027 \pm 0,01^{\mathrm{c}}$ & $0,04 \pm 0,009^{c}$ & $1,7 \pm 0,9^{c}$ & $0,05 \pm 0,01^{\mathrm{ab}}$ \\
\hline $30 / 25$ & $12 / 12$ & $0,02 \pm 0,01^{\mathrm{bc}}$ & $0,05 \pm 0,03^{c}$ & $2,8 \pm 1,0^{\mathrm{bc}}$ & $0,06 \pm 0,02^{\mathrm{ab}}$ \\
\hline $30 / 25$ & $14 / 10$ & $0,009 \pm 0,003^{a}$ & $0,03 \pm 0,01^{\mathrm{bc}}$ & $3,7 \pm 1,0^{\mathrm{ab}}$ & $0,06 \pm 0,02^{\mathrm{ab}}$ \\
\hline
\end{tabular}

Médias nas colunas seguidas pela mesma letra não diferem estatisticamente ao nível de $5 \%$ de probabilidade pelo teste de Tukey $(\mathrm{n}=5)$.

O efeito conjugado da temperatura e do fotoperíodo também influenciaram no desempenho fotossintético de P. pectinatus. Valores de Pmax foram crescentes com o aumento do fotoperíodo em temperaturas de crescimento baixas $\left(10^{\circ} \mathrm{CD} / 05^{\circ} \mathrm{CN}\right)$ e decrescentes nas temperaturas altas $\left(30^{\circ} \mathrm{CD} / 25^{\circ} \mathrm{CN}\right.$ ) (Tabela 2 , Figura 3). Menores valores foram observados nas temperaturas de $30^{\circ} \mathrm{CD} / 25^{\circ} \mathrm{CN}$ e maiores valores nas de $20^{\circ} \mathrm{CD} / 15^{\circ} \mathrm{C}$ (Tabela 2 , Figura 3). O maior valor de a foi observado em plantas crescendo em temperaturas de $20^{\circ} \mathrm{CD} / 15^{\circ} \mathrm{CN}$, fotoperíodo de $10 \mathrm{hL} / 14 \mathrm{hE}\left(\mathrm{a}=0,92 \pm 0,16 \mathrm{gO}_{2} \mathrm{gMS}^{-1} \mathrm{~h}^{-1} / \mathrm{mmol}\right.$ $\mathrm{m}^{-2} \mathrm{~s}^{-1}$ ) (Tabela 2). Maior valor de Ic $\left(98,77 \pm 0,05 \mathrm{mmol} \mathrm{m}^{-2} \mathrm{~s}^{-1}\right)$ foi observado na temperatura de $30^{\circ} \mathrm{CD} / 25^{\circ} \mathrm{CN}$, fotoperíodo $14 \mathrm{hL} /$ $10 \mathrm{hE}$, e o menor valor de Ic $\left(5,88 \pm 0,18 \mathrm{mmol} \mathrm{m}^{-}\right.$ ${ }^{2} \mathrm{~s}^{-1}$ ) em $10^{\circ} \mathrm{CD} / 05^{\circ} \mathrm{CN}$, fotoperíodo $14 \mathrm{hL} / 10 \mathrm{hE}$ (Tabela 1). Maior Ik $\left(281,75 \pm 10,75 \mathrm{mmol} \mathrm{m}^{-2} \mathrm{~s}^{-1}\right)$ foi observado na temperatura $30^{\circ} \mathrm{CD} / 25^{\circ} \mathrm{CN}$, fotoperíodo $12 \mathrm{hL} / 12 \mathrm{hE}(\mathrm{p}<0,05)$, e menor $\mathrm{Ik}$ $\left(34,14 \pm 0,69 \mathrm{mmol} \mathrm{m} \mathrm{m}^{-2} \mathrm{~s}^{-1}\right.$ em $10^{\circ} \mathrm{CD} / 05^{\circ} \mathrm{CN}$, fotoperíodo $14 \mathrm{hL} / 10 \mathrm{hE}$ (Tabela 2 ). A respiração não apresentou diferença significativa $(p>0,05)$ entre as plantas crescendo nas diferentes temperaturas e fotoperíodos (Tabela 2). 
Efeito da temperatura e do fotoperíodo no crescimento e nas respostas fotossintéticas de Potamogeton pectinatus L. (Potamogetonaceae), em cultivo experimental

TABELA 2 - Parâmetros das curvas PI em folhas de Potamogeton pectinatus crescendo em diferentes temperaturas e fotoperíodos. $\alpha=$ inclinação inicial da curva $\left(\mathrm{gO}_{2} \mathrm{gMS}^{-1} \mathrm{~h}^{-1} / \mathrm{mmol} \mathrm{m}^{-2} \mathrm{~s}^{-1}\right)$. Ic $=$ radiação de compensação $\left(\mathrm{mmol} \mathrm{m} \mathrm{s}^{-1}\right)$. Pmax $=$ fotossíntese líquida máxima $\left(\mathrm{gO}_{2} \mathrm{gMS}^{-1} \mathrm{~h}^{-1}\right)$. Ik $=$ radiação de saturação $\left(\mathrm{mmol} \mathrm{m}^{-2} \mathrm{~s}^{-1}\right)$.

Respiração $\left(\mathrm{gO}_{2} \mathrm{gMS}^{-1} \mathrm{~h}^{-1}\right) \cdot(\mathrm{n}=4)$

\begin{tabular}{ccccccc}
\hline Temp. & Fotop. & $\alpha$ & Ic & Pmax & Ik & Respiração \\
\hline $10 / 05$ & $10 / 14$ & $0,73 \pm 0,23^{\mathrm{a}}$ & $11,31 \pm 0,07^{\mathrm{a}}$ & $0,43 \pm 0,19^{\mathrm{a}}$ & $47,03 \pm 1,21^{\mathrm{ab}}$ & $0,18 \pm 0,17^{\mathrm{a}}$ \\
$10 / 05$ & $12 / 12$ & $0,64 \pm 0,24^{\mathrm{a}}$ & $8,85 \pm 0,13^{\mathrm{a}}$ & $0,49 \pm 0,15^{\mathrm{a}}$ & $34,98 \pm 0,85^{\mathrm{b}}$ & $0,27 \pm 0,19^{\mathrm{a}}$ \\
$10 / 05$ & $14 / 10$ & $0,78 \pm 0,31^{\mathrm{a}}$ & $5,88 \pm 0,18^{\mathrm{a}}$ & $0,50 \pm 0,22^{\mathrm{a}}$ & $34,14 \pm 0,69^{\mathrm{b}}$ & $0,25 \pm 0,08^{\mathrm{a}}$ \\
$20 / 15$ & $10 / 14$ & $0,92 \pm 0,16^{\mathrm{a}}$ & $16,70 \pm 0,05^{\mathrm{ac}}$ & $0,53 \pm 0,12^{\mathrm{a}}$ & $50,28 \pm 1,0^{\mathrm{a}}$ & $0,25 \pm 0,10^{\mathrm{a}}$ \\
$20 / 15$ & $12 / 12$ & $0,23 \pm 0,37^{\mathrm{b}}$ & $62,17 \pm 0,14^{\mathrm{b}}$ & $0,34 \pm 0,07^{\mathrm{ab}}$ & $123,72 \pm 2,90^{\mathrm{c}}$ & $0,34 \pm 0,24^{\mathrm{a}}$ \\
$20 / 15$ & $14 / 10$ & $0,48 \pm 0,28^{\mathrm{ab}}$ & $24,65 \pm 0,28^{\mathrm{bc}}$ & $0,57 \pm 0,23^{\mathrm{a}}$ & $47,48 \pm 0,64^{\mathrm{a}}$ & $0,41 \pm 0,39^{\mathrm{a}}$ \\
$30 / 25$ & $10 / 14$ & $0,32 \pm 0,36^{\mathrm{b}}$ & $27,69 \pm 0,06^{\mathrm{c}}$ & $0,28 \pm 0,2^{\mathrm{ab}}$ & $116,24 \pm 5,07^{\mathrm{c}}$ & $0,06 \pm 0,03^{\mathrm{a}}$ \\
$30 / 25$ & $12 / 12$ & $0,13 \pm 0,44^{\mathrm{b}}$ & $97,54 \pm 0,09^{\mathrm{d}}$ & $0,27 \pm 0,15^{\mathrm{ab}}$ & $281,75 \pm 10,75^{\mathrm{d}}$ & $0,16 \pm 0,07^{\mathrm{a}}$ \\
$30 / 25$ & $14 / 10$ & $0,16 \pm 0,33^{\mathrm{b}}$ & $98,77 \pm 0,05^{\mathrm{d}}$ & $0,19 \pm 0,05^{\mathrm{b}}$ & $237,06 \pm 11,48^{\mathrm{d}}$ & $0,15 \pm 0,13^{\mathrm{a}}$ \\
\hline
\end{tabular}

Médias nas colunas seguidas pela mesma letra não diferem estatisticamente ao nível de $5 \%$ de probabilidade pelo teste de Tukey $(\mathrm{n}=5)$.

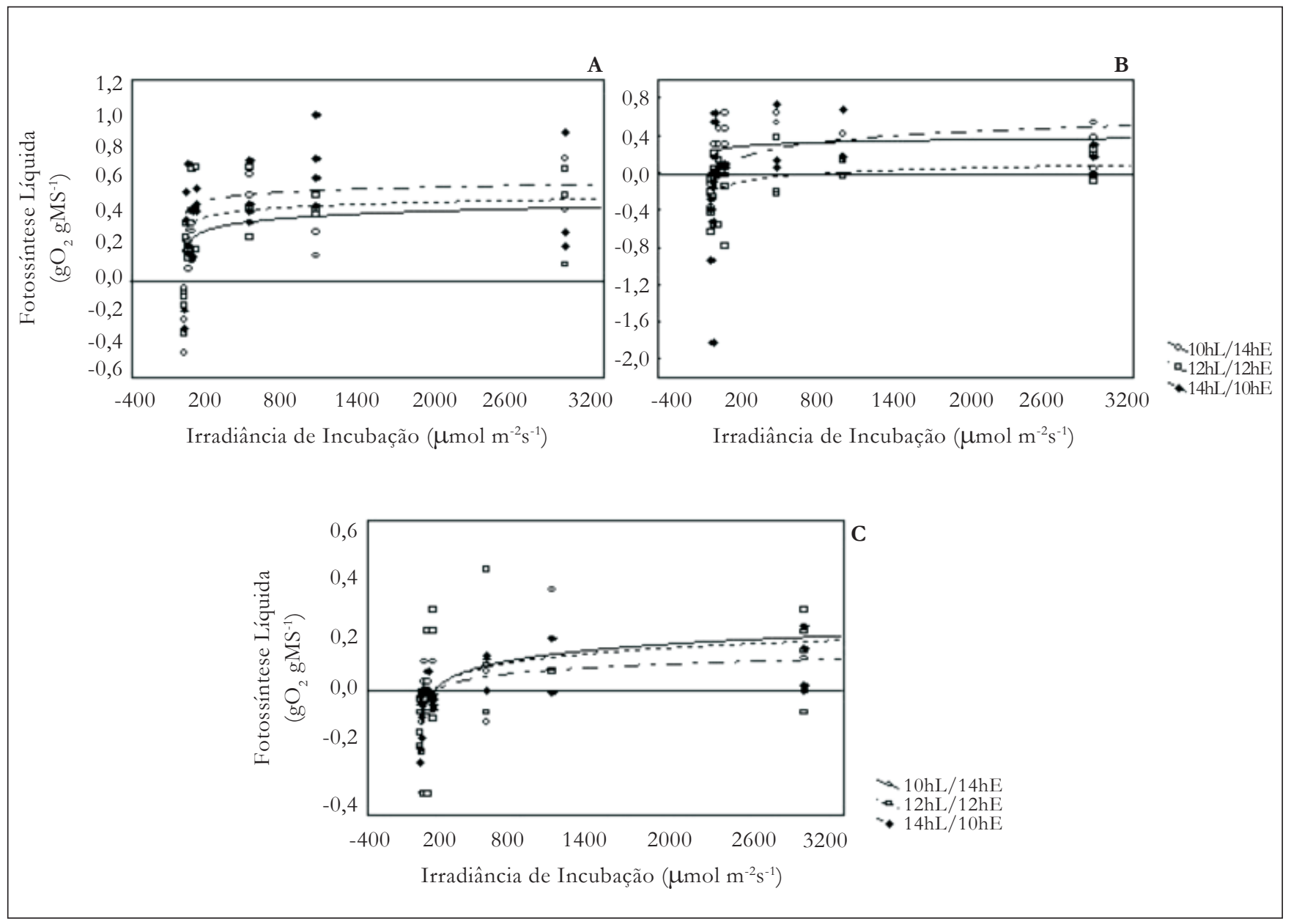

FIGURA 3 - Curvas PI (Fotossíntese XIrradiância) das folhas de Potamogetonpectinatus L., crescendo em cultivo experimental em diferentes temperaturas e fotoperíodos.

Nota: Temperaturas de incubação: A) $10^{\circ} \mathrm{C}$; B) $20^{\circ} \mathrm{C}$; C) $30^{\circ} \mathrm{C}$ 


\section{DISCUSSÃO}

A capacidade de aclimatação das espécies às variações na temperatura pode aumentar sua produtividade (9). A temperatura da água pode influenciar a produtividade das plantas aquáticas levando as espécies há apresentar ajustes fisiológicos, morfológicos ou de alocação de biomassa, em resposta a variações termais $(8,25)$. As plantas de $P$. pectinatus utilizadas neste estudo, responderam com ajustes morfológicos ou fisiológicos as temperaturas e fotoperíodos testados. Maior produção, melhor desenvolvimento e maior longevidade foliar foram observados nas temperaturas maiores. O desempenho fotossintético aumentou proporcionalmente ao tempo de exposição à luz quando em temperaturas baixas, enquanto que nas temperaturas elevadas, menor tempo de exposição à luz estimulou fisiologicamente as plantas, resultando num maior Pmax.

Temperaturas baixas e fotoperíodos curtos interferem no comprimento final alcançado pelas folhas alterando sua biomassa (25). Temperaturas altas e fotoperíodos prolongados implicam excesso de radiação, levando a inibição do crescimento, por danos nos tecidos fotossintéticos (26). O efeito conjugado temperatura baixa $\mathrm{x}$ fotoperíodo curto simulou uma situação de inverno, desfavorável ao crescimento das plantas, que apresentaram tendência a alongar as folhas já existentes, com menor investimento na produção de novas folhas e raízes. Por sua vez, na temperatura alta, um fotoperíodo curto foi suficiente para estimular a produção de tecidos fotossintéticos garantindo a captação mais eficiente da luz. Esses resultados estão de acordo com o observado por Colares et al. (12), reforçando a adaptação da espécie às temperaturas de verão, com investimento em crescimento horizontal, visando maior ocupação e exploração do espaço quando em condições ótimas de temperatura.

Em regiões temperadas, menores temperaturas coincidem com baixas irradiâncias e curtos fotoperíodos, o que induz uma aclimatação à temperatura e luz associadas (25). Os efeitos dos dois parâmetros são difíceis de serem isolados uma vez que a temperatura da água é determinada pela quantidade de radiação solar incidente (27). $O$ aumento na temperatura de incubação de $20^{\circ} \mathrm{C}$ para $30^{\circ} \mathrm{C}$ resultou em queda na produção fotossintética (Pmax), com valores de a baixos. Valores de a representam o incremento da taxa fotossintética com aumento da irradiância, estando relacionados às reações luminosas da fotossíntese, que são independentes da temperatura (28). Essa redução no Pmax e a, nas maiores temperaturas, pode estar indicando temperatura acima do ótimo da espécie. Essa situação é ainda reforçada pelos maiores valores de Ic e Ik observados, e podem estarmostrando uma resposta adaptativa à temperatura média anual da Lagoa Verde $\left(22,4 \pm 2,8^{\circ} \mathrm{C}\right)$, onde as plantas foram coletadas (12).

A respiração e o desempenho fotossintético podem estar associados à idade das folhas (29). Em temperaturas de crescimento e incubação iguais, a aclimatação pode conduzir a taxas de respiração variáveis (8). Neste estudo,P.pectinatus não apresentou diferença significativa nos valores respiratórios nas diferentes temperaturas e fotoperíodos testados, apesar da utilização de folhas de idades similares. Macrófitas aquáticas possuem a capacidade de estocar oxigênio no espaço aéreo lacunar e com isto apresentar diferenças entre o oxigênio dissolvido e as taxas de respiração (10). Desta forma, acredita-se que os valores de respiração observados não estejam relacionados à idade da folha, nem adaptação à temperatura, mais sim a utilização do oxigênio dos espaços aerequimatosos da planta, mascarando a quantidade de $\mathrm{O}_{2}$ utilizado na respiração, não apresentando diferenças na taxa de oxigênio dissolvido na água.

Variações na temperatura podem alterar a produção de folhas, sem interferir na biomassa final das plantas aquáticas. A biomassa final alcançada é balanceada pela produção de folhas e pelo aumento em espessura e comprimento dos pecíolos em Potamogeton nodosus (30) e em Nelumbo lutea (31). Segundo esses autores, essas espécies respondem melhor a temperatura do que a luz, alongando os pecíolos sem alterar a biomassa. Em nosso estudo, apesar da diferente produção de folhas e de raízes, em função da temperatura e fotoperíodo, a biomassa final alcançada pelas plantas não variou. Provavelmente, as plantas de $P$. pectinatus crescendo em diferentes regimes de temperaturas alcançaram as mesmas biomassas finais por mecanismos compensatórios de produção ou alongamento de folhas e/ou pecíolos, como observado por Al-Hamdani e Francko (30).

Trocas nas condições de crescimento, com alocação de biomassa aérea em subterrânea, como resposta à exposição a elevadas temperaturas são observadas em macrófitas submersas (17). Reservas energéticas são estocadas para serem utilizadas durante os períodos de baixas temperaturas de inverno $(31,32)$. Populações de P. pectinatus na Lagoa Verde apresentam esse mecanismo de alocação de 
biomassa aérea em subterrânea como estratégia para sobreviver nas baixas temperaturas de inverno, reiniciando o brotamento na primavera, quando as condições de luz e temperatura são mais apropriadas ao crescimento (12). Neste estudo, a relação biomassa subterrânea / aérea de $P$. pectinatus foi sempre maior nas temperaturas maiores, o que pode estar indicando um acúmulo de reservas nutritivas nos tecidos subterrâneos. Isto também pode ser visualizado pela taxa de crescimento específico, onde apesar do maior investimento na produção de folhas na temperatura de $30^{\circ} \mathrm{CD} / 25^{\circ} \mathrm{CN}$, a taxa de crescimento específico foliar, expressa por biomassa, foi menor. Provavelmente, a menor taxa de crescimento específico observada nas maiores temperaturas seja decorrente da alocação da biomassa para os órgãos subterrâneos, uma vez que não foram observadas variações significativas na biomassa final.

A capacidade de aclimatação das espécies as variações de temperatura podem levar a diferenças sazonais na capacidade fotossintética e consequentemente no crescimento e biomassa. Populações de P. pectinatus na Lagoa Verde apresentam um ciclo de vida sazonal controlado por fatores abióticos (12). As respostas morfológicas e fisiológicas observadas neste estudo demonstram a plasticidade morfológica de $P$. pectinatus para se adaptar as diferentes condições sazonais, características de regiões de clima temperado, com melhor crescimento em temperaturas amenas de verão.

\section{AGRADECIMENTOS}

Os autores agradecem ao Instituto de Ciências Biológicas (ICB) da Universidade Federal do Rio Grande (FURG) pelo apoio técnico; à Fundação de Assistência à Pesquisa do Estado do Rio Grande doSul(FAPERGS), pelo financiamento do projeto; ao Conselho Nacional de Desenvolvimento Científico e Tecnológico (CNPq) pela concessão da Bolsa de Iniciação Científica.

\section{REFERÊNCIAS}

1. Monson RK, Littlejohn RO, Williams GJ. Photosynthectic adaption to temperature in four species from the Colorado shortgrass steppe: a physiological model for coexistence. Oecologia. 1983;58(1):43-51.
2. Reeves PH, Coupland G. Response of plant development to environment: control of flowering by daylenght and temperature. Current Opinion in Plant Biology. 2000;3(1):37-42.

3. Sand-Jensen K, Madsen TV. Minimum light requirements of submerged freshwater macrophytes in laboratory growth experiments. Journal of Ecology. 1991;79(3):749-64.

4. Evans AS, Webb KL, Penhale PA. Phoosynthetic temperature acclimation in two coexisting seagrasses, Zostera marina L. and Ruppia maritima L. Aquatic Botany. 1986;24(2):185-97.

5. Petrucio MM, Esteves FA. Influence of photoperiod on the uptake of nitrogen and phosphorus in the water by Eichhornia crassipes and Salvinia auriculata. Rev Bras Biol. 2000;60(3):373-379.

6. Palma-Silva C, Albertoni EF, Esteves FA. Clear water associated with biomass and nutrient variation during the growth of a Charophyte stand after a drawdown, in a tropical coastal lagoon. Hydrobiologia. 2002;482:79-87.

7. Pilon J, Santamaría L. Clonal variation in morphological and physiological responses to irradiance and photoperiod for the aquatic angiosperm Potamogeton pectinatus. Journal of Ecology. 2002;90(5):859-70.

8. Berry JA, Björkman O. Photosynthetic response and adaptation to temperature in higher plants. Annual Review of plant physiology. 1980;31:491-543.

9. Van der Bijl L, Sand-Jensen K, Hjermind AL. Photosyntesis and canopy structure of a submerged plant, Potamogeton pectinatus, in a danish lowland stream. Journal of Ecology. 1989;77(4):947-62.

10. Pilon J, Santamaría L. Clonal variation in the thermal responses of the submerged aquatic macrophyte Potamogeton pectinatus. Journal of Ecology. 2002;90(1):141-152.

11. Carr GM, Duthie HC, Taylor WD. Models of aquatic plant productivity: a review of the factors that influence growth. Aquatic Botany. 1997;59(3):195-215. 
12. Colares IG, Schlee MDB, Santos LC, Magalhães UAS. Variação da biomassa e produtividade de Potamogeton pectinatus L. (Potamogetonaceae) na Lagoa Verde, Rio Grande, RS. Iheringia, Série Botânica. 2007;62(1/2):131-7.

13. Chadin I, Volodin V, Whiting P, Shirshova T, Kolegova N, Dinan L. Ecdysteroid contend and distribution in plants of genus Potamogeton. Biochemical Systematics. 2003;31(4):407-15.

14. Dixon MH, Hill SA, Jackson MB, Ratcliffe RG, Sweetlove LJ. Physiological and metabolic adaptations of Potamogeton pectinatus L. tubers support rapid elongation of stem tissue in the absence of oxygen. Plant Cell Physiology. 2006;47(1):128-40.

15. Schoschina EV. Seasonal and age dynamics of growth and reproduction of Phycodrys rubens (Rhodophyta) in the Barents and White Seas. Aquatic Botany. 1996;55(1):13-30.

16. Hawes I, Sutherland D, Hanelt D. The use of pulse amplitude modulated fluorometry to determine fine-scale temporal and spatial variation of insitu photosynthetic activity within an Isoetes-dominated canopy. Aquatic botany. 2003;77(1):1-15.

17. Olesen B, Sand-Jensen K. Seasonal acclimatization of eelgrass Zostera marina growth to light. Marine Ecology Progress Series. 1993;94:91-99.

18. Colares IG, Seeliger U. Influência da luz sobre o crescimento e a produção de biomassa de Ruppia maritima L. em cultivo experimental. Acta Botanica Brasílica. 2006;20(1):31-36.

19. Strickland JDH, Parsons TR. A practical handbook of seawater analysis. Bulletin of the Fisheries Research Board of Canada. 1972;167:185-99.

20. Kirk JTO. Light and photosyntesis in aquatic ecosystems. 2nd ed. Cambridge: Cambridge University Press; 1994.

21. Goodman JL, Moore KA, Denninson WC. Photosynthetic responses of eelgrass (Zostera marina L.) to light and sediment sulfide in a shallow barrier island lagoon. Aquatic Botany. 1995;50(1):37-47.

22. Masini RJ, Cary JL, Simpson CJ, Mccomb AJ. Effects of light and temperature on the photosynthesis of temperature meadowforming seagrass in Western Australia. Aquatic Botany. 1995;49:239-254.
23. Menendez M, Sanchez A. Seasonal variations in P-I responses of Chara hispida L. and Potamogeton pectinatus L. from stream mediterranean ponds. Aquatic Botany.1998;61(1):1-15.

24. Zar JH. Biostatistical analysis. 2nd ed. New Jersey: Prentice-Hall, Inc; 1984.

25. Ludlow MM. Light stress at high temperature. In: Kyle DJ, Osmond CB, Arntzen CJ, editor. Photoinhibition (9). 2nd ed. Amsterdam (The Netherlands): Elsevier science publishers B.V. Biomedical Division; 1987. p. 90-107.

26. Salisbury FB, Ross CW Plant physiology. 4th ed. California: Wadsworth Publishing Company; 1992.

27. Colares IG. Influência da luz sobre o crescimento e fotossíntese de Ruppia maritima L., no estuário da Lagoa dos Patos, RS [tese]. Rio Grande: Universidade Federal do Rio Grande; 1998.

28. Santamaría L, Hootsmans MJM. The effect of temperature on the photosyntesis, growth and reproduction of a Mediterranean submerged macrophyte, Ruppia drepanensis. Aquatic Botany. 1998;60:169-88.

29. Barko DG, Hardin DG, Mattews MS. Growth and morphology of submerged freshwater macrophytas in relatin to light and temperature. Canadian Journal Botany. 1982;60:877-87.

30. Al-Hamdani S, Francko DA. Effect of light and temperature on photosynthesis, elongation rate and chlorophyll content of Nelumbo lutea (willd.) Pers. Seedlings. Aquatic Botany. 1992;44(1):51-58.

31. Jian Y, Li B, Wang J, Chen J. Control of turion germination in Potamogeton crispus. Aquatic Botany. 2003;75(1):59-69.

32. Santamaria L, Garcia AIL. Latitudinal variation in tuber production in an aquatic pseudo-annual plant Potamogeton pectinatus. Aquatic Botany. 2004;79:51-64.

Recebido: 07/07/2007

Received: 07/07/2007

Aprovado: 05/11/2007

Approved: 11/05/2007 